\title{
Fitzpatrick Skin Type VI
}

National Cancer Institute

\section{Source}

National Cancer Institute. Fitzpatrick Skin Type VI. NCI Thesaurus. Code C74574.

Skin which is dark brown or black and it never burns when exposed to the sun. 